\title{
colaboraciones
}

\section{HORMIGONES LIGEROS Y OBRAS PUBLICAS FRANCIA}

G. Vié, Ingeniero de Minas

683-6

\section{RESUMEN}

Entre las causas del empleo, cada vez más amplio, del hormigón ligero frente al hormigón tradicional se encuentran: menor peso, menor contracción durante el fraguado, transporte menos costoso, colocación y manejo más fácil, etc.

En este artículo se describe la realización de una pasarela en Grenoble (Francia), de 125,70 m de longitud, de los cuales 68,80 son de hormigón ligero.

El tablero, de una anchura de 6,7 $m$ de longitud y pretensado longitudinalmente, está formado por una viga-cajón de $1,5 \mathrm{~m}$ de altura e inercia constante. La cimentación se realizó sobre pilotes de 800 a $1.000 \mathrm{~mm}$ de diámetro prefabricados in situ. Los estribos son del tipo contrapeso.

La pasarela se construyó en dos mitades, cada una de ellas sobre una orilla del río Isère, uniéndose posteriormente una vez colocadas en su emplazamiento final.

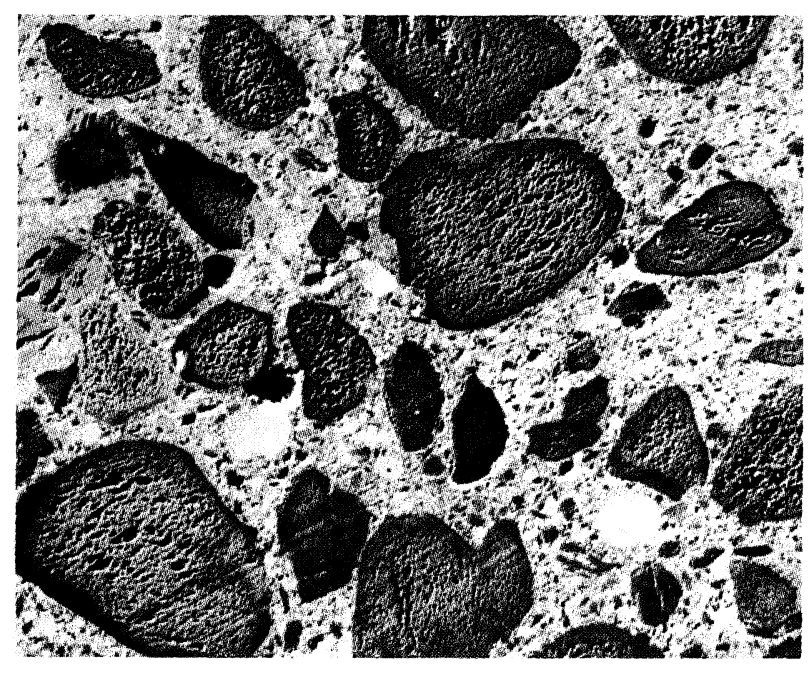

Corte de una probeta de hormigón ligero (ampliada cuatro veces I.
En el número 284 (octubre 1976) de INFORMES, se publico un primer artículo sobre "Utilizaciones de los esquistos carboníferos en la construcción", con descripción del procedimiento de fabricación de dichos áridos.

En el presente artículo se describe una ampliación particular de un hormigón a base de áridos ligeros. Como introducción conviene recordar algunos puntos con ellos relacionados:

\section{Hormigones ligeros}

En esta clase se incluyen los hormigones destinados a ser armados o pretensados, es decir, las vigas, postes, losas, pantallas, etc., además de los muros y paramentos.

Es evidente que cuanto más ligera es una viga menos tiene que "soportar". Una economía del orden del $30 \%$ en el peso del hormigón, da una ganancia de aproximadamente el $30 \%$ sobre las armaduras y las cimentaciones. Por ello, la tendencia futura será a recurrir cada vez menos al hormigón tradicional, con áridos extraidos del lecho de los ríos o de las rocas de las canteras.

El empleo del hormigón ligero resulta mucho más interesante cuando se aplica a estructuras en las que la importancia del peso propio es preponderante. La experiencia demuestra que construyendo un puente con hormigon ligero, se reduce en varios puntos (por ejemplo $4 \%$ ) el precio de la obra. Por otra parte, los áridos ligeros actuales absorben mucha menos agua, lo cual conlleva una menor contracción en el momento del fraguado. Incluso en la construccion de edificios, los hormigones ligeros facilitan la prefabricación de los elementos, que resultan más fáciles de mantener y de colocar y cuyo transporte es menos costoso. En la restauración de viviendas antiguas, el hormigón ligero permite no aportar sobrecargas a estructuras antiguas.

El agotamiento lento pero inevitable de los recursos de áridos tradicionales, las exigencias de la ecologia, el incremento de los precios de los áridos clásicos contribuirán a poner fin, antes de lo que se creia, a su uso cotidiano.

Aparte de las consideraciones relacionadas con el medio ambiente, hay que tener en cuenta la mejora que representa, en el plano económico, la utilización de los materiales expandidos, ya que para 


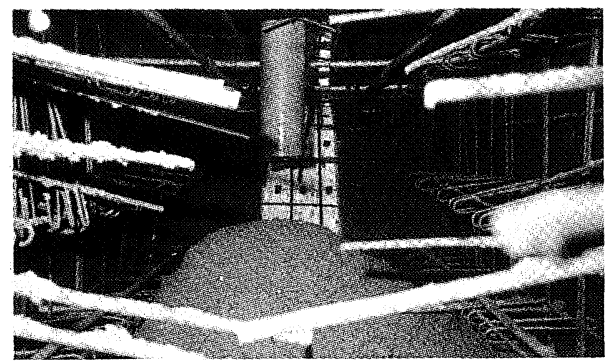

Pila de la orilla izquierda durante el hormigonado.

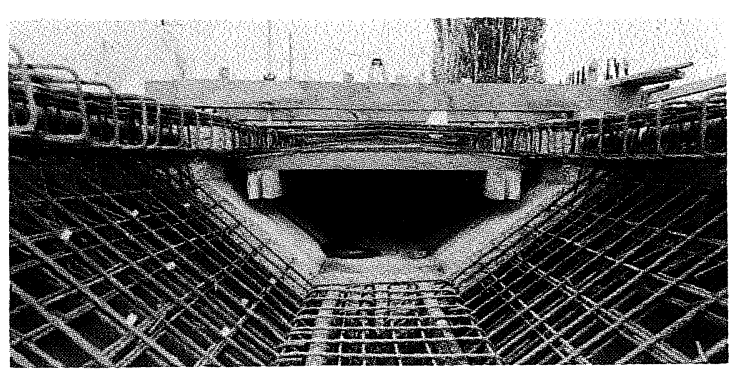

Encofrado y armadura del cordón inferior de la dovela del tablero.

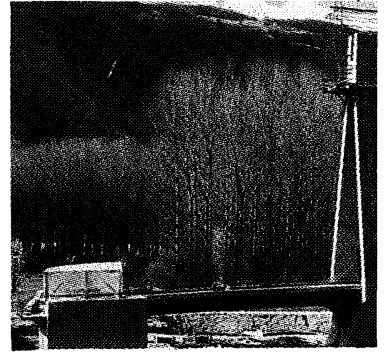

Semiestructura de obra en una orill un mismo volumen de hormigón se necesita a menudo una cantidad tres o cuatro veces menor.

La primera aplicación del hormigón de áridos ligeros se remonta a comienzos de siglo, cuando se construyo un barco de carga de 7.500 toneladas en los Estados Unidos, al que se bautizo con el nombre de "SELMA".

La primera vez que se utilizo el hormigón ligero para la construcción de una obra pública, fue en el puente colgante "Golden Gate" en San Francisco, durante el año 1932. Esta nueva tecnología fue adaptada posteriormente en Europa, hacia 1960, principalmente para diversas obras en Alemania y en los Países Bajos. Francia realizo su primera experiencia con el puente de Cheneau (departamento de Vosges), en 1971. A este puente siguieron otras aplicaciones, y desde 1979 Francia ostenta un récord europeo y otro mundial que son, respectivamente: el puente de TRICASTIN (Vaucluse), con tablero de $142,5 \mathrm{~m}$ de hormigón ligero y el puente de OTTMARSHEIM (Alto Rhin), con $338 \mathrm{~m}$ de luz total, de los que $171 \mathrm{~m}$ son de hormigon ligero.

Estas diversas realizaciones facilitaron y alentaron la concepción de obras todavía más audaces, Ilevadas a cabo con un dominio perfecto, entre las que se encuentra la construcción de una pasarela sobre el Isère en GRENOBLE, realizada en 19801981. Como indica el esquema, la longitud total de esta reciente obra es de $125,70 \mathrm{~m}$, con un vano central de $79 \mathrm{~m}$ de luz, de los que $68,80 \mathrm{~m}$ son de hormigón ligero. La particularidad esencial de esta pasarela consiste en haber sido concebida y construida como estructura atirantada, en hormigón ligero.

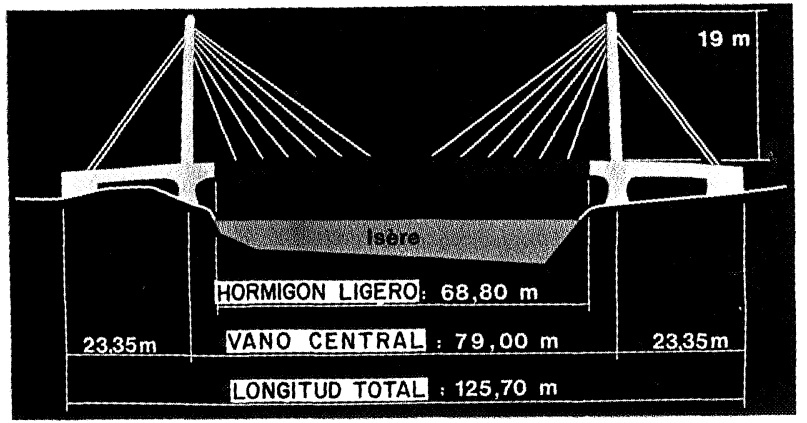

Esquema de la pasarela de hormigón ligero sobre el rio lsère.
El material utilizado para la preparación de los áridos ligeros es un esquisto bituminoso cribado cuya densidad es de $2.700 \mathrm{~kg}$ por metro cúbico. Al sufrir un choque térmico a $1.150{ }^{\circ} \mathrm{C}$ de temperatura este esquisto se expande para engendrar áridos mucho menos pesados, con lo que su densidad se reduce a $1.200 \mathrm{~kg} / \mathrm{m}^{3}$, o sea, un $55 \%$ con relación a la roca natural de origen.

Es notable señalar que el hormigón obtenido a partir de estos áridos de esquisto expandido, of rece caracteristicas bastante similares a las del hormigón clásico, con una densidad evidentemente mucho menor.

En la preparación del hormigón, a partir de la dosificacion de sus constituyentes, para que se obtengan las excelentes características mecánicas que más adelante se mencionan, deben respetarse unas estrictas y reducidas tolerancias.

Insistimos sobre todo en la dosificación del agua de amasado del hormigón, ya que los áridos expandidos son ávidos de agua y pueden absorber fácilmente más de la necesaria (coeficiente másico de fijación de agua para estos áridos): por ejemplo, $6 \%$ a las dos horas y $8 \%$ a las cuarenta y ocho horas).

\begin{tabular}{|c|c|c|}
\hline & $\begin{array}{l}\text { Hormigón } \\
\text { tradicional }\end{array}$ & $\begin{array}{c}\text { Hormigón } \\
\text { ligero }\end{array}$ \\
\hline Densidad & 2,4 & 1,8 \\
\hline Resistencia a la compresión a los 28 dias & $50 \mathrm{MPa}$ & $40 \mathrm{MPa}$ \\
\hline Resistencia a la tracción a los 28 días & $4,5 \mathrm{MPa}$ & $3 \mathrm{MPa}$ \\
\hline $\begin{array}{l}\text { Retracción final } \\
\text { (Unidad arbitraria) }\end{array}$ & 1 & 1,5 \\
\hline Coeficiente de fluencia & 2 & 1.2 a 1.8 \\
\hline
\end{tabular}

\section{Estudio de proyecto de una pasarela en Grenoble}

La ciudad de Grenoble ha tenido un desarrollo considerable en el curso de estos treinta últimos años. El urbanismo, la industrialización, el crecimiento de los medios universitarios modificaron completamente el aspecto de esta ciudad. 

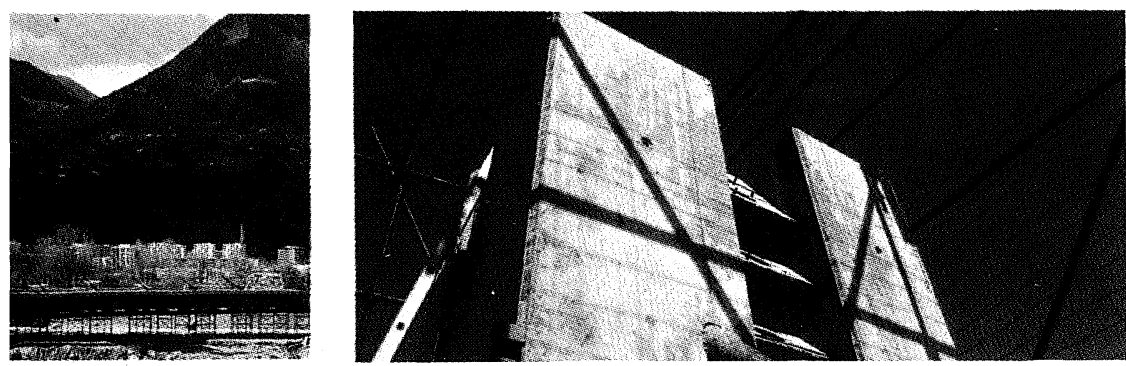

Detalle de pilono: anclajes de tirantes.

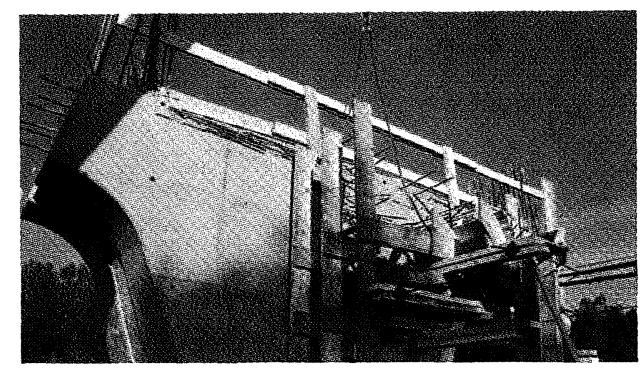

Pila de la orilla derecha desencofrada.
Pionera en la utilización de los grandes saltos y de las fuerzas hidráulicas naturales, esta ciudad de montañas se convirtio, con sus 400.000 habitantes, en la primera ciudad técnica y científica de provincia. Su asombrosa diversificación industrial explica esta expansión excepcional.

Dos importantes ríos, el Isère y el Drac, atraviesan la ciudad y, a pesar de la proliferación de puentes, los itinerarios continuaban siendo demasiado largos y molestos.

Al noroeste de la ciudad, la situación es todavía más crítica para los peatones y vehículos de dos ruedas, que no pueden circular por la Carretera Nacional $n .^{\circ} 90$, reservada exclusivamente a la circulación de automóviles. Para llegar a la zona universitaria, antes era necesario dar un largo rodeo por el puente denominado de Sablons, lo que representa un recorrido de casi $4.500 \mathrm{~m}$ para una distancia real tres veces menor.

Ante estas dificultades, surgio la idea de una estructura, lo más simple posible, para peatones y vehículos de dos ruedas, que pasara por encima del Isère, entre los barrios de Meylan y de Saint Martín d'Heres, en la cercania del sector universitario. La búsqueda de una inversión reducida y el deseo de respetar la estética de un lugar muy hermoso hicieron que la Administracion (en este caso el Municipio de Meylan) prefiriese una solución que no se apoyase en el río.

Debido a ello, se penso en construir la obra sobre las orillas del Isère y luego colocar el tablero por rotación.

Teniendo en cuenta la importancia de la masa que habia que levantar y girar, la Dirección Departamental de Infraestructura, la Dirección de Obras y las Oficinas de estudio y de asistencia técnica, se esforzaron en reducir al máximo el peso de la obra, recurriendo para ello al hormigón ligero que, como ya se ha dicho, tiene un $30 \%$ de ganancia en peso con relación al hormigón clásico. También se busco una disminución en el peso del conjunto, reduciendo la altura del tablero.

Para conseguir una esbeltez de 0,019 (relación entre el espesor de tablero y su longitud) era necesario el empleo de un arriostramiento; por consi- guiente, se construyo una pasarela con alambres tensores de hormigón ligero colocada por rotacion.

\section{Características principales de la obra}

Como ya se ha indicado antes, la longitud total de la pasarela es de $125,70 \mathrm{~m}$, con un vano central de $79 \mathrm{~m}$ de los que $68,8 \mathrm{~m}$ están hechos de hormigón ligero. Los dos vanos de las orillas tienen una longitud de 23,35 m cada uno.

La cimentación se hizo sobre pilotes de 800 y $1.000 \mathrm{~mm}$ de $\varnothing$, prefabricados in situ y con una profundidad de $22 \mathrm{~m}$ por debajo del terreno natural, por lo general aluvial, de las riberas del Isère. Las pilas son de hormigón armado, con una armadura de 3,8 t de acero por pila.

Un pretensado transversal, aplicado a nivel del tablero por seis barras Dywidag, retoma los esfuerzos de tracción que resultan de la forma particular del pilono.

El hormigón se fabricó con cemento CPA 55, dosificado a $350 \mathrm{~kg} / \mathrm{m}^{3}$. Mientras que la pila situada en la orilla izquierda se retaco, la de la orilla derecha reposa sobre cuatro juntas de neopreno.

La altura de los pilonos es de $19 \mathrm{~m}$ con una disposicion en dos ramas que se unen perpendicularmente a los bloques de anclaje de los cables de arriostramiento.

El tablero tiene las siguientes características:

- Viga-cajón de inercia constante de altura = $=1,50 \mathrm{~m}$.

- Ancho de 6,7 m que comprende una acera y una pista para vehículos de dos ruedas, con separación asegurada por los anclajes de los cables tirantes.

- El pretensado es longitudinal.

Los estribos son del tipo contrapeso: el de la orilla derecha descansa sobre tres juntas de neopreno $500 \times 500$, mientras que el de la orilla izquierda y el encepado correspondiente están solidarizados. 


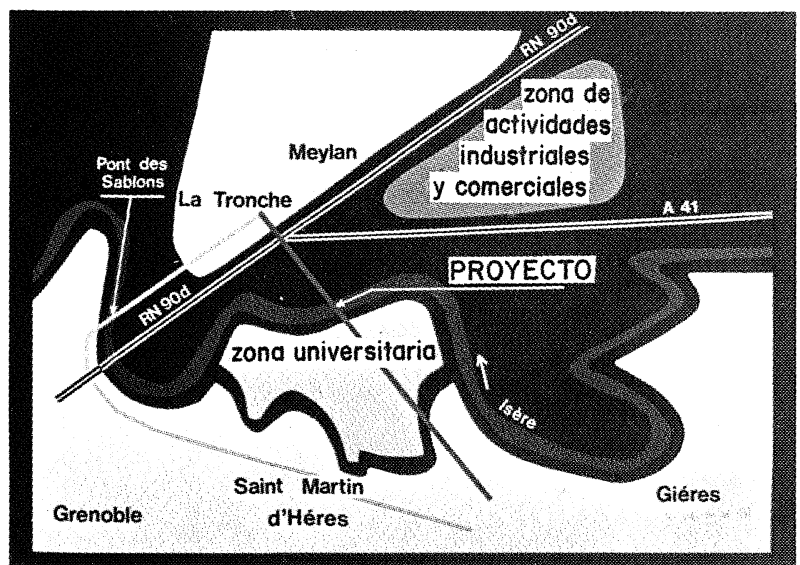

Ubicación de la obra en fase de estudio.

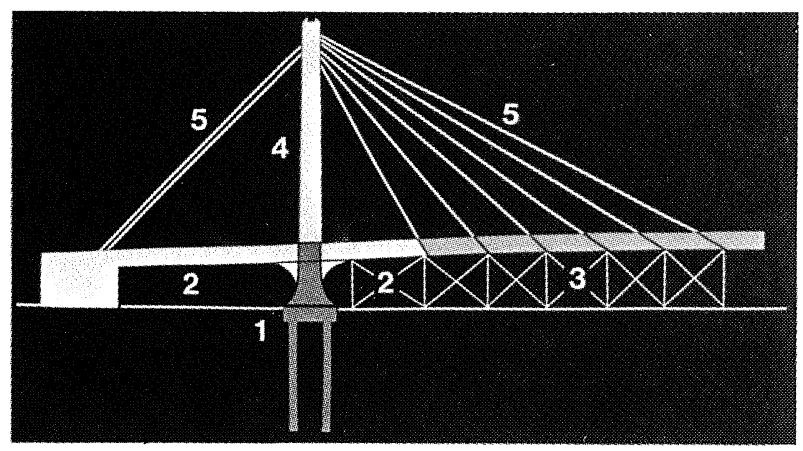

Fases sucesivas de la construcción:

1. - Perforación y hormigonado de cimientos sobre pilotes y construcción de la pila.

2. - Hormigonado clásico del vano extremo y la primera dovela del tramo central.

3. - Hormigonado de dovelas.

4. - Construcción de pilonos.

5. - Colocación de tirantes.

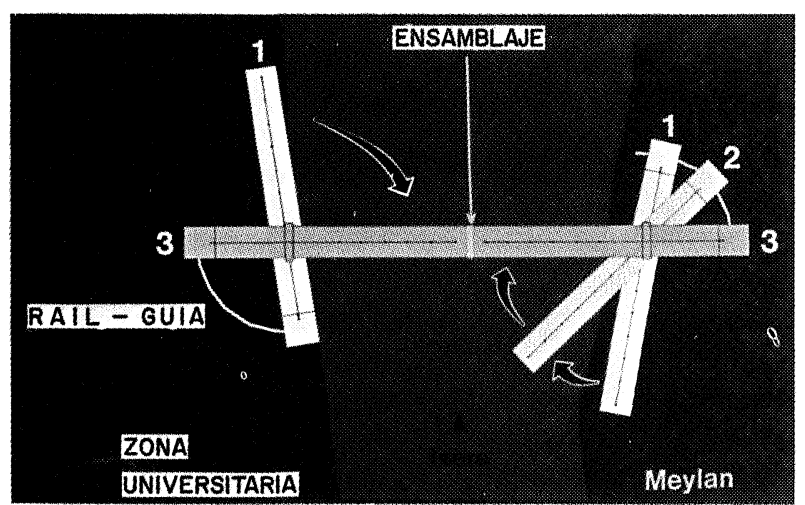

Proceso de montaje:

1. - Semiestructura en construcción sobre la simbra.

2. - Semiestructura en posición intermedia.

3. - Semiestructura en posición definitiva.

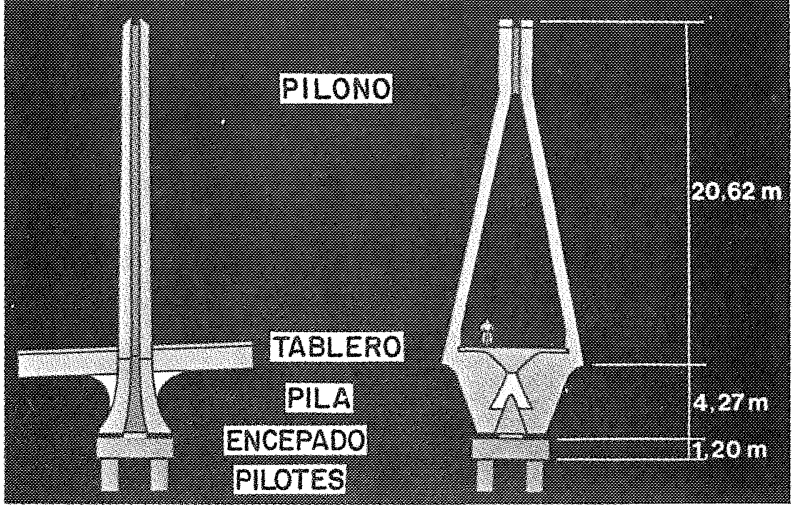

Esquema de los pilonos.

El sistema de suspensión, consta por una parte, de 6 cables tirantes 12 t 15 por pilares, repartidos sobre el eje del tramo central y, por otra, de 2 cables tirantes 19 t 15 de reacción por pilono, anclados sobre los estribos. Existe un dispositivo de amortiguamiento hidráulico de las vibraciones, realizado con un aceite de fuerte viscosidad introducido en las vainas de acero de los cables tirantes.

La barandilla tipo S 8 es de acero galvanizado pintado, revestido con un listón de madera.

\section{Diversas fases de la construcción}

Esta estructura, que comprende un vano central y dos adyacentes, se ha construido, sobre cimbra, en dos mitades, en cada una de las dos orillas del río Isère y paralelamente a éste.

Cinco fases esenciales han permitido la construcción de cada mitad:

1. ${ }^{\circ}$ Perforación y hormigonado de los cimientos sobre pilotes y construcción de la pila.

2. Construcción en hormigón clásico del vano extremo y de la primera dovela del vano central.

3. Realización, en hormigón ligero, de las demás dovelas.

4. ${ }^{\circ}$ Construcción de cada pilono.

5. Colocación de los cables tirantes.

La puesta bajo tensión de éstos provocó la desolidarizacion del tablero y de la cimbra, lo que hizo posible la colocación de la estructura, por rotación de cada una de ambas mitades. 


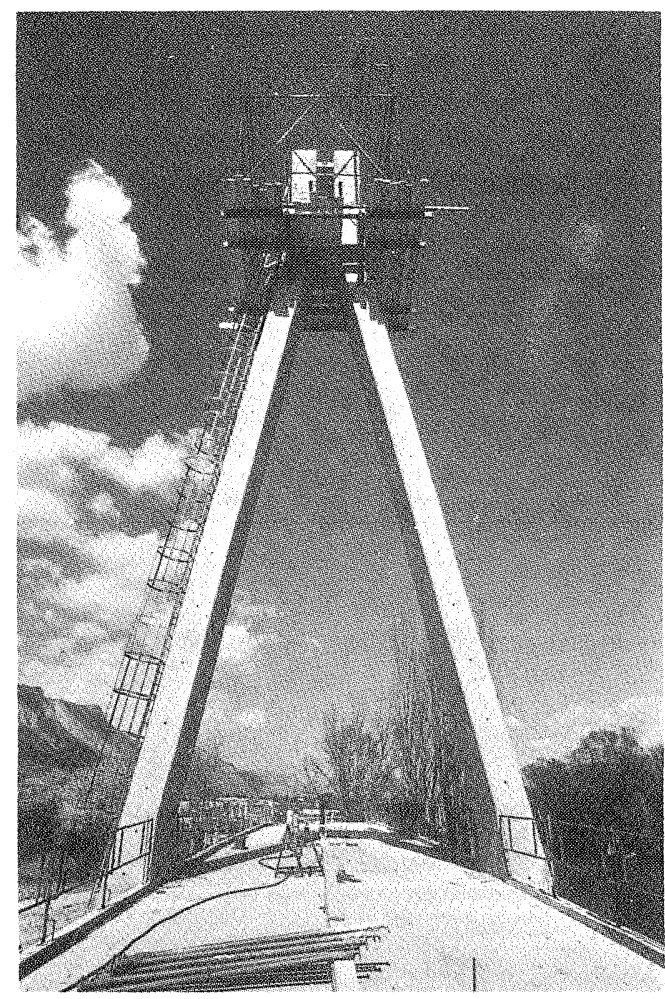

Pilono después del desencofrado.

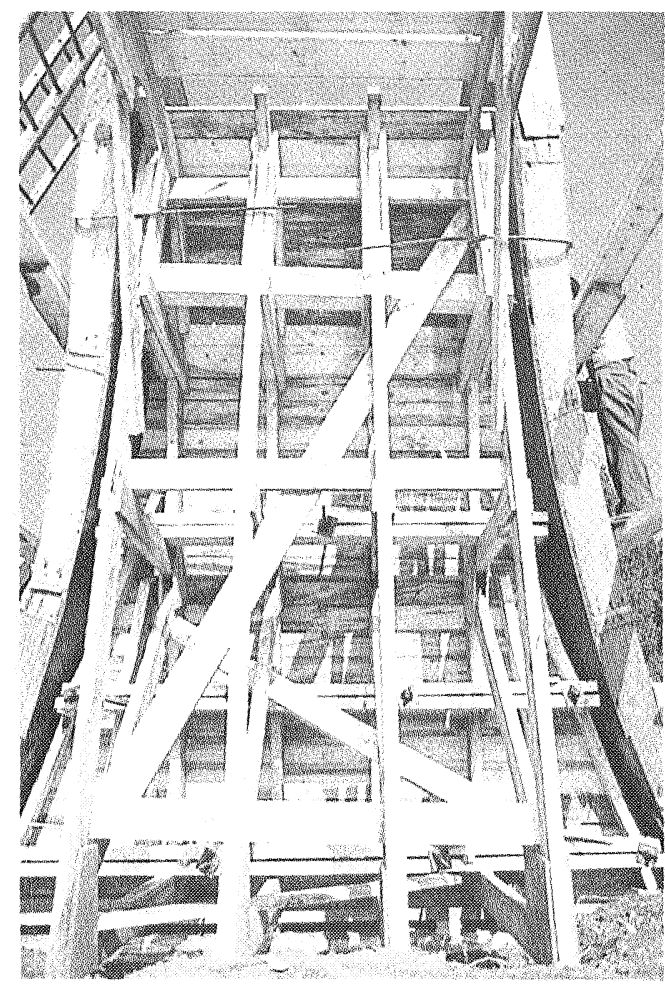

Encofrado de la pila de la orilla derecha.
Encofrado del pilono.

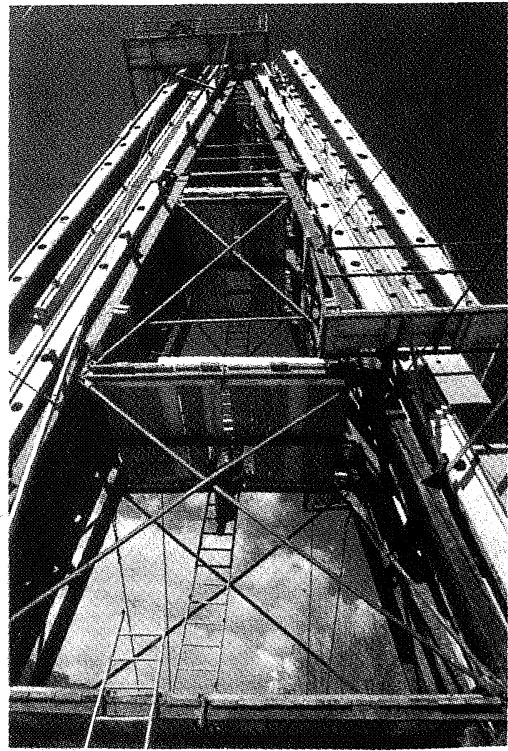

El proceso puede resumirse de este modo:

Cada mitad, se levanto por medio de gatos hidráulicos. La rotación se realizó con un ángulo proximo a $90^{\circ}$, hasta alcanzar la posición final, por encima del río.

Los estribos contrapeso, cuyo relleno solo se realizo hasta la mitad con el fin de reducir el peso de las partes móviles, se desplazaron a lo largo de rieles-guía sobre rodillos.

Una vez colocadas de modo definitivo las dos partes de la obra, se procedio a la unión de las dos partes de la pasarela. En cuanto se acabó esta fase, se construyeron las placas de apoyo de caucho artificial neopreno de la orilla derecha.

Las placas de apoyo de la orilla izquierda se suprimieron sustituyéndose por un recalce de la pila sobre la zapata de la cimentación.

Esta solidarización entre tablero y cimientos tiene como finalidad reducir las vibraciones susceptibles de producirse por el efecto de vientos bastante fuertes. Las vibraciones residuales se admiten pues no tienen mayores consecuencias para la estabilidad de la pasarela.

Al finalizar las diversas fases, se procedio a los ensayos bajo cargas rodantes, mediante varios camiones de 3,5 t de peso unitario, en movimiento o parados sobre el tablero.

Aunque esta obra solo está prevista para el paso de peatones, con o sin sus bicicletas, los ensayos se hicieron pensando en un excepcional paso de vehículos de motor, que tuvieran que franquearlo por razones urgentes de seguridad y de socorro. 